\title{
Vaporization of heated materials into discharge plasmas
}

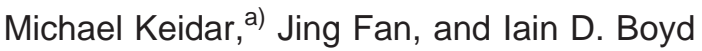 \\ Department of Aerospace Engineering, University of Michigan, Ann Arbor, Michigan 48109 \\ Isak I. Beilis \\ Electrical Discharge and Plasma Laboratory, Fleischman Faculty of Engineering, Tel Aviv University, \\ P.O.B. 39040, Tel Aviv 69978, Israel
}

(Received 28 August 2000; accepted for publication 12 December 2000)

\begin{abstract}
The vaporization of condensed materials in contact with high-current discharge plasmas is considered. A kinetic numerical method named direct simulation Monte Carlo (DSMC) and analytical kinetic approaches based on the bimodal distribution function approximation are employed. The solution of the kinetic layer problem depends upon the velocity at the outer boundary of the kinetic layer which varies from very small, corresponding to the high-density plasma near the evaporated surface, up to the sound speed, corresponding to evaporation into vacuum. The heavy particles density and temperature at the kinetic and hydrodynamic layer interface were obtained by the analytical method while DSMC calculation makes it possible to obtain the evolution of the particle distribution function within the kinetic layer and the layer thickness. (C) 2001 American Institute of Physics. [DOI: 10.1063/1.1345860]
\end{abstract}

\section{INTRODUCTION}

The vaporization of a heated surface interacting with discharge plasmas has a great interest for different applications such as ablation controlled arcs, ${ }^{1}$ pulsed plasma thrusters, ${ }^{2,3}$ high-pressure discharges, ${ }^{4}$ vacuum arcs,${ }^{5}$ electroguns, ${ }^{6}$ and metal evaporation by laser radiation action. ${ }^{7}$ In most models, the rate of evaporation is calculated using the Langmuir relationship ${ }^{8}$ that is, however, limited to the case of vaporization into vacuum.

Anisimov ${ }^{9}$ considered a case of vaporization of a metal exposed to laser radiation using a bimodal velocity distribution function in the nonequilibrium (kinetic) layer. The main result of this work is the calculation of the maximal flux of returned atoms to the surface, which was found to be about $18 \%$ of the flux of vaporized atoms. This result was obtained under the assumption that the atom flow velocity is equal to the sound velocity at the external boundary of the kinetic layer. In many physical situations, however, the expansion of the vapor is not by the sound speed since there is a dense plasma in the volume discharge. Beilis ${ }^{10,11}$ analyzed metal vaporization into discharge plasmas in the case of a vacuum arc cathode spot. He concluded that the parameters at the outer boundary of the kinetic layer are close to their equilibrium values and that the velocity at the outer boundary of the kinetic layer is much smaller than the sound velocity. In both the analyses mentioned above, no information is provided about the change of the particle velocity distribution function from a nonequilibrium state to an equilibrium state inside the kinetic layer.

In the present article we study the nonequilibrium layer close to the evaporating surface using the particle method known as direct simulation Monte Carlo (DSMC). ${ }^{12}$ It will determine the thickness of the nonequilibrium layer and the

${ }^{\text {a)} E l e c t r o n i c ~ m a i l: ~ k e i d a r @ e n g i n . u m i c h . e d u ~}$ evolution of the particle distribution function within the layer. The numerical simulation results will be compared with the analytical results for the case when the vapor velocity at the outer boundary of the kinetic layer is given as a parameter.

\section{MODEL OF THE NONEQUILIBRIUM LAYER}

In this section we will present two different kinetic models (particle simulation and analytical approach) for the nonequilibrium layer near the evaporating surface.

\section{A. Particle simulation}

In the nonequilibrium layer near the surface there are collisions between particles that eventually lead to a change of the distribution function. The DSMC method uses particle motion and collisions to perform a simulation of gas dynamics under nonequilibrium conditions. Each particle has spatial and velocity coordinates. The collision approach between particles is based on a probability model developed from the kinetic theory and commonly used in DSMC. ${ }^{12}$

To perform the DSMC simulation we have to specify conditions at two boundaries (see Fig. 1). At the evaporating surface with density $n_{0}$ and temperature $T_{0}$, the velocity distribution function for emitted particles is in the equilibrium form $^{9,10}$

$$
f_{0}(\mathbf{V})=n_{0}\left(\frac{m}{2 \pi k T_{0}}\right)^{3 / 2} \exp \left(-\frac{m V^{2}}{2 k T_{0}}\right), \quad V_{x}>0 .
$$

At the outer boundary of the kinetic layer the distribution function for particles is assumed to be

$$
f_{1}(\mathbf{V})=n_{1}\left(\frac{m}{2 \pi k T_{1}}\right)^{3 / 2} \exp \left(-m \frac{\left(V_{x}-V_{1}\right)^{2}+V_{y}^{2}+V_{z}^{2}}{2 k T_{1}}\right),
$$




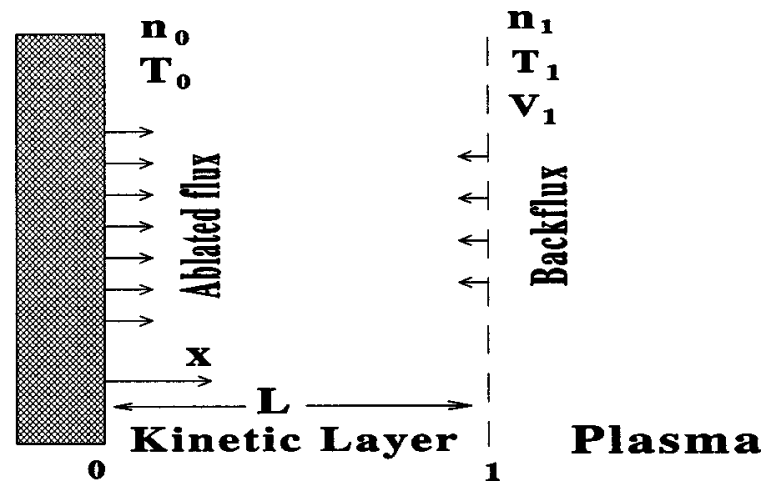

FIG. 1. Schematic representation of the near surface layer.

where $V_{1}$ is the velocity, $n_{1}$ is the density, and $T_{1}$ is the temperature. These boundary conditions are supplemented by using an empirical relation between $T_{0}$ and $n_{0}$. As an example, we have used the equilibrium vapor pressure for the case of Teflon in the form ${ }^{2}$

$$
P_{0}=P_{c} \exp \left(-T_{c} / T_{0}\right) \text { and } n_{0}=P_{0} / k T_{0},
$$

where $P_{0}$ is the equilibrium pressure, $P_{c}=1.84 \times 10^{20} \mathrm{~N} / \mathrm{m}^{2}$ and $T_{c}=20815 \mathrm{~K}$ are the characteristic pressure and temperature obtained empirically. The physical meaning of the characteristic pressure and temperature is that the equilibrium pressure equals $P_{c}$ when vapor reaches the temperature of $T_{c}$. The calculations are performed assuming that vapor consists of $\mathrm{CF}_{2}$ molecules at a surface temperature in the range $550-650 \mathrm{~K}$ that is typical for an electrothermal pulsed plasma thruster. ${ }^{3}$

The DSMC model employed in the present article has the following strategy. Uniform cells $0.5 \lambda$ in size are employed, and time step $\Delta t=0.3 \lambda / V_{m, s}$ (Ref. 13) where $\lambda$ and $V_{m, s}=\left(2 k T_{0} / m\right)^{0.5}$ are the molecular mean free path and the most probable thermal speed at the ablated surface. Molecules enter the flow field successively from the surface due to evaporation, and from the outer boundary due to Maxwellian velocity distribution that allows particle velocities in the negative direction. Using the assumption about the distribution function at the surface and at the external boundary Eqs. (1) and (2) we can calculate the flux of molecules entering from the surface, $G_{s}$, and from the outer boundary of the layer, $G_{b}$, as follows:

$$
G_{s}=n_{0}\left(2 k T_{0} / m\right)^{0.5} / 2 \pi^{0.5}
$$

and

$$
\begin{aligned}
G_{b}= & n_{1}\left(2 k T_{1} / m\right)^{0.5} \\
& \times\left[\exp \left(-\alpha^{2}\right)-\pi^{0.5} \alpha\{1-\operatorname{erf}(\alpha)\}\right] / 2 \pi^{0.5},
\end{aligned}
$$

where $\alpha=V_{1} /\left(2 k T_{1} / m\right)^{0.5}$. The molecular interaction is described by the variable hard-sphere (VHS) model. ${ }^{12}$ The VHS model is employed to select molecular collision pairs from cells and to distribute the postcollision velocities. This model assumes that the scattering from molecular collision is isotropic in the center of mass frame of reference. ${ }^{12}$ Both boundaries (wall and outer boundary of the kinetic layer) are assumed to be perfectly absorbing. The flow will arrive at a steady state when the sum of $G_{s}$ and $G_{b}$ is exactly balanced by the flux of molecules leaving from the outer boundary or sticking on the surface:

$$
G_{s}+G_{b}=G_{r}+G_{f}
$$

where $G_{r}$ is the flux of the particles returned to the surface during the time step and $G_{f}$ is the flux of the particles crossing the outer boundary of the layer.

In the DSMC approach, in order to calculate the evolution of the distribution function inside the kinetic layer, we have to specify the thickness of the layer in units of $\lambda$. The parameters at the outer boundary of the kinetic layer $\left(n_{1}\right.$ and $T_{1}$ ) and the flux of returned particles are calculated as a function of the distance of the location of the outer boundary of the layer and of the velocity at this boundary $V_{1}$.

\section{B. Analytical approach}

Let us consider the analytical approach developed in Refs. 9-11, where the vapor parameters $T_{1}$ and $n_{1}$ at the outer boundary can be obtained without information about the layer thickness. This means that the problem is reduced to integration of the conservation equations of mass, momentum, and energy across the layer. ${ }^{9}$ We consider a nonequilibrium layer (thickness of about a mean free path $\lambda$ ) adjacent to the surface (as shown in Fig. 1), where the velocity distribution function of the evaporated molecules reached equilibrium by the rare-field collisions with the background heavy particles and furthermore the vapor flow is described by a hydrodynamic approach. Using Anisimov's assumption ${ }^{9}$ that the velocity distribution function for the returned particles $\left(V_{x}<0\right)$ is $\beta f_{1}(\mathbf{V})$, where $\beta$ is the proportionality coefficient, the relation of the heavy particle parameters at the outer boundary of the kinetic layer in the case of an arbitrary velocity is obtained from the model ${ }^{10}$ and reads as follows:

$$
\begin{aligned}
\frac{n_{0}}{2\left(\pi d_{0}\right)^{0.5}}= & n_{1} V_{1}+\beta \frac{n_{1}}{2\left(\pi d_{1}\right)^{0.5}} \\
& \times\left\{\exp \left(-\alpha^{2}\right)-\alpha \pi^{0.5} \operatorname{erfc}(\alpha)\right\},
\end{aligned}
$$

$$
\begin{aligned}
\frac{n_{0}}{4 d_{0}}= & \frac{n_{1}}{2 d_{1}}\left\{\left(1+2 \alpha^{2}\right)\right. \\
& \left.-\beta\left[\left(0.5+\alpha^{2}\right) \operatorname{erfc}(\alpha)-\alpha \exp \left(-\alpha^{2}\right) / \pi^{0.5}\right]\right\},
\end{aligned}
$$

$$
\begin{aligned}
\frac{n_{0}}{\left(\pi d_{0}\right)^{1.5}}= & \frac{n_{1}}{\left(d_{1}\right)^{1.5}} \pi^{-1}\left[\alpha\left(\alpha^{2}+2.5\right)-0.5 \beta\left\{\left(2.5+\alpha^{2}\right) \alpha\right.\right. \\
& \left.\left.\times \operatorname{erfc}(\alpha)-\left(2+\alpha^{2}\right) \exp \left(-\alpha^{2}\right) / \pi^{0.5}\right\}\right]
\end{aligned}
$$

where $d_{0}=m / 2 k T_{0}, d_{1}=m / 2 k T_{1}, \operatorname{erfc}(\alpha)=1-\operatorname{erf}(\alpha)$, and $\operatorname{erf}(\alpha)$ is the error function. The equation system (7) is obtained using the boundary conditions (1)-(3) and the conservation laws of mass, momentum, and energy across the layer. ${ }^{9,10}$ By calculating the parameters at the outer boundary of the kinetic layer we can obtain the flux of returned particles: 


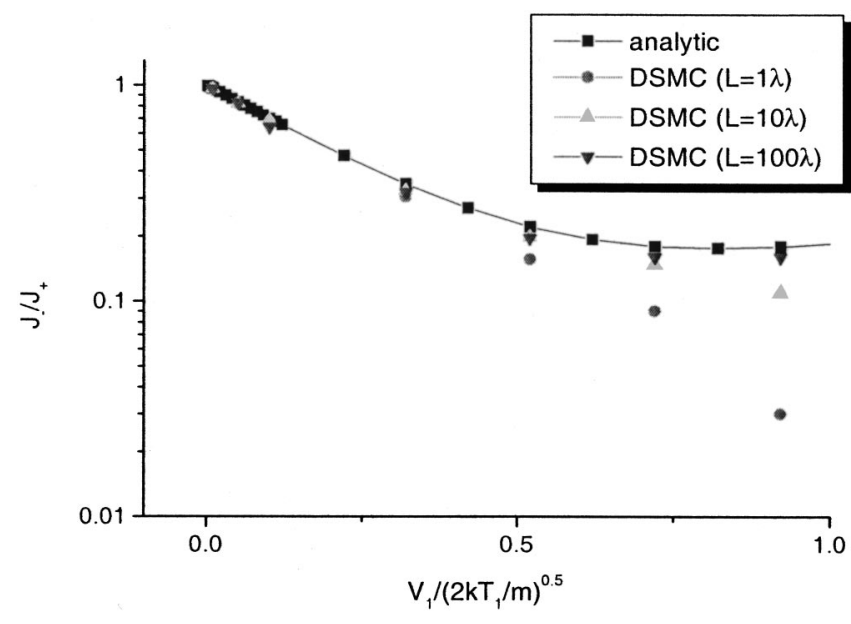

FIG. 2. Comparison of the analytic and DSMC return flux as a function of velocity $V_{1}$ with the distance of location of the outer boundary of the kinetic layer $L$ as a parameter.

$$
\begin{aligned}
J_{-} & =\int_{-\infty}^{0} \beta f_{1}(V) V d V \\
& =\beta N_{1}\left(\frac{k T_{1}}{2 \pi m}\right)^{0.5}\left\{\exp \left(-\alpha^{2}\right)-\alpha \pi^{0.5} \operatorname{erfc}(\alpha)\right\} .
\end{aligned}
$$

The system of equations (7) has four unknowns and therefore the solution can be found having one unknown as a parameter, which is the velocity $V_{1}$ at the outer boundary of the kinetic layer in our case.

\section{RESULTS}

DSMC calculations and a comparison with the analytical predictions for the flux of returned atoms is shown in Fig. 2 where the thickness of the kinetic layer is used as a parameter. One can see that in the case of small velocity ( $\alpha$ $\leqslant 0.5$ ) at the outer boundary all results agree well. This is the case when the thickness of the nonequilibrium layer is about one mean free path. In the case when evaporation occurs at about the sound velocity at the outer boundary, the DSMC calculations approach the analytical value at a layer thickness of $\sim 10-20$ mean free paths.

The calculation of the backflux dependence with distance inside the layer when $\alpha=1$ is presented in Fig. 3. It can be seen that in the case when $V_{1}$ is about the sound velocity, the flux of the returned molecules depends upon the distance from the evaporating surface where the external boundary is placed. Thus up to a layer thickness of about 20 mean free paths, the flux changed strongly and then it is weakly saturated. The DSMC calculation predicts a $16 \%$ flux of returned particles, which is very close to the analytical result of $18 \%$. The reason for this difference can be understood by analyzing the velocity distribution function of returned particles in the DSMC calculation.

Results of the DSMC calculation of the velocity distribution function and comparison with the analytic approximation $\beta f_{1}(\mathbf{V})$ are shown in Fig. 4(a) for the case of sound velocity at the outer boundary. One can see that the distribution functions remain different in the case of a $100 \lambda$ kinetic

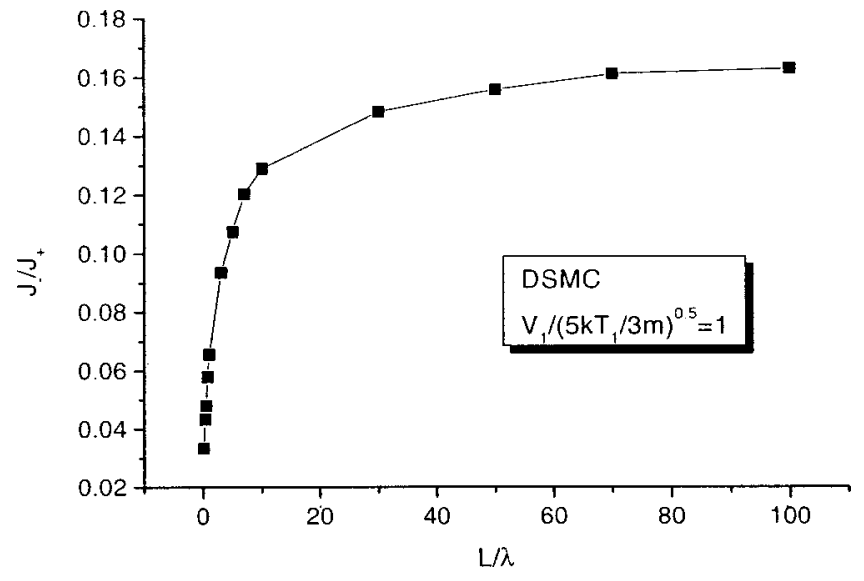

FIG. 3. The DSMC calculated return flux as a function of the distance of location of the outer boundary of the kinetic layer $L$ in the case of sound velocity at the boundary 1 .

layer thickness. This is not the case when the velocity at the outer boundary of the kinetic layer is small as shown in Fig. 4(b), where the DSMC distribution function agrees well with the analytic approximation. Therefore it is not surprising that the calculated flux of returned particles is also found to be in good agreement with the analytical result. It should be noted
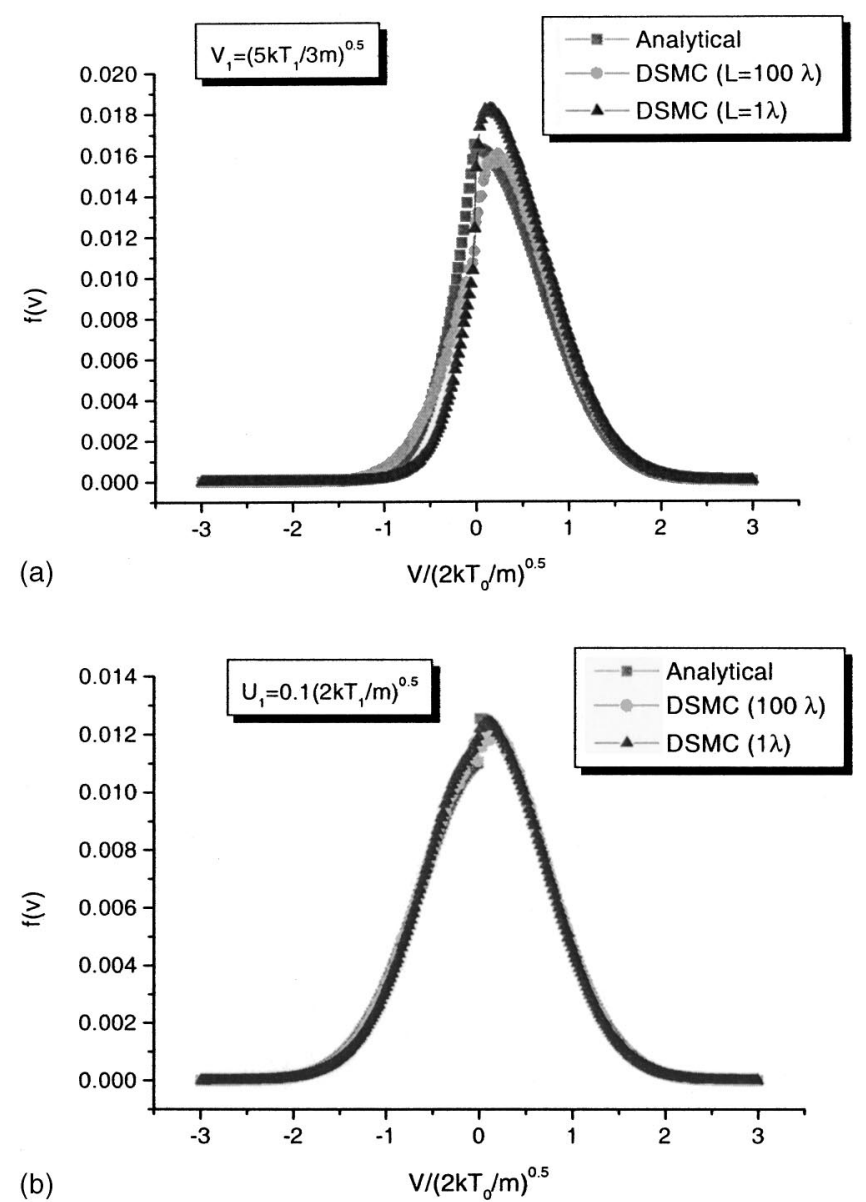

FIG. 4. Variation of the velocity distribution function of the returned particles near the wall with the distance of location of the outer boundary of the kinetic layer $L$ as a parameter. (a) $V_{1}=\left(5 k T_{1} / 3 m\right)^{0.5}$ and (b) $V_{1}$ $=0.1\left(2 k T_{1} / m\right)^{0.5}$. 


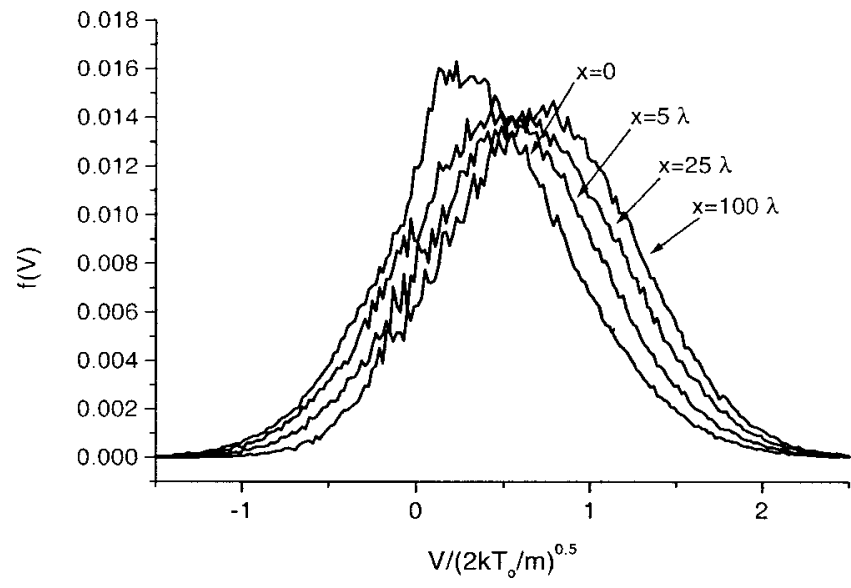

FIG. 5. Variation of the velocity distribution function (normal to the surface component) with the distance from the evaporated surface as a parameter. The thickness of the kinetic layer $L=100 \lambda$ and $V_{1}=\left(5 k T_{1} / 3 m\right)^{0.5}$.

that the discontinuity in the analytical distribution function [Fig. 4(b)] is the result of the definition of the distribution function of the returned particles (Sec. II B).

The evolution of the particle distribution function within the Knudsen layer is shown in Fig. 5 for the case $V_{1}$ $=\left(5 k T_{1} / 3 m\right)^{0.5}$. One can see that the velocity distribution function approaches a drifted Maxwellian at a distance of several mean free paths from the surface. The drift velocity slightly increases with further distance from the evaporating surface.

The results of calculation of the analytic system of equations (7) are presented in Fig. 6 with the normalized velocity $V_{1}$ as a parameter. The temperature $T_{1}$, density $n_{1}$, and the flux of returned particles $J_{-}$all decrease as the velocity at the outer boundary of the kinetic layer increases. In the limiting case of the sound velocity, the flux of returned particles is equal to $18 \%$ as was obtained by Anisimov. ${ }^{9}$ In this case the analytically predicted flux of returned particles is larger than that obtained by numerical simulations (16\%, see Fig. 3 ). It should be pointed out that the dependence of the flux of

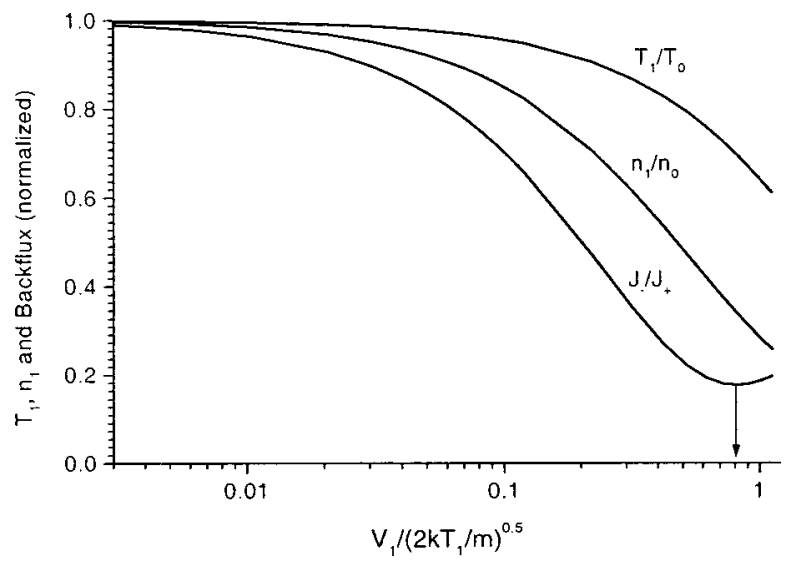

FIG. 6. Parameters (temperature, density, and returned flux) at the outer boundary of the kinetic layer as a function of velocity $V_{1}$ calculated from Eq. (7). returned particles $J_{-}$on the velocity $V_{1}$ has a minimum near the sound speed (see Fig. 6). The minimum corresponds to the sound speed with adiabatic index 1.3. This fact as well as the overestimate of the returned particle flux is connected with the assumption in the analytical approach of the form of the particle velocity distribution for the particles returned to the evaporating surface, i.e., that the distribution function of the returned particles is proportional to the distribution function at the outer boundary of the kinetic layer. ${ }^{9}$

\section{SUMMARY}

Two kinetic approaches, namely the particle method (DSMC) and bimodal distribution function approach were employed to describe the parameters in the nonequilibrium kinetic layer near the evaporating surface. DSMC calculation makes it possible to establish the thickness of the kinetic layer and the evolution of the particle distribution function within the layer. The thickness of the kinetic layer and the vapor density and temperature in the kinetic layer adjacent to the evaporating surface depend upon the velocity at the outer boundary of the layer. We have found that the thickness of the kinetic layer increases from a few mean free paths $\lambda$ in the case of small velocity up to about 10-20 $\lambda$ in the case of the evaporation with sound speed at the outer boundary of the layer. Comparison of the DSMC and analytical results indicates that the analytical model predicts correctly the flux of returned particles over a wide range of velocity at the outer boundary of the layer. The present model can be used for calculation of the rate of evaporation of the heated surface interacting with a plasma. The free parameter of this model, the velocity at the outer boundary of the layer, can be determined by coupling this model with a model of the hydrodynamic layer and the plasma bulk.

\section{ACKNOWLEDGMENT}

M.K., J.F., and I.D.B. gratefully acknowledge the financial support of the Air Force Office of Scientific Research through Grant No. F49620-99-1-0040.

${ }^{1}$ C. B. Ruchti and L. Niemeyer, IEEE Trans. Plasma Sci. 14, 423 (1986).

${ }^{2}$ R. Burton and P. Turchi, J. Propul. Power 14, 716 (1998).

${ }^{3}$ M. Keidar, I. D. Boyd, and I. I. Beilis, IEEE Trans. Plasma Sci. 28, 376 (2000).

${ }^{4}$ M. I. Boulos, P. Fauchais, and E. Pfender, Thermal Plasmas: Fundamentals and Applications, Vol. 1 (Plenum, New York, 1995).

${ }^{5}$ Vacuum Arc Science and Technology, edited by R. L. Boxman, P. Martin, and D. Sanders (Noyes, Park Ridge, NJ, 1995).

${ }^{6}$ L. L. Raja, P. L. Varghese, and D. E. Wilson, J. Thermophys. Heat Transfer 11, 353 (1997).

${ }^{7}$ L. V. Zhigilei, P. B. S. Kodali, and B. J. Garrison, J. Phys. Chem. B 102, 2845 (1998).

${ }^{8}$ I. Langmuir, Phys. Rev. 2, 329 (1913).

${ }^{9}$ S. I. Anisimov, Sov. Phys. JETP 27, 182 (1968).

${ }^{10}$ I. I. Beilis, IEEE Trans. Plasma Sci. 13, 288 (1985).

${ }^{11}$ I. I. Beilis, Theoretical Modeling of Cathode Spot Phenomena, in Vacuum Arc Science and Technology, edited by R. L. Boxman, P. Martin, and D. Sanders (Noyes, Park Ridge, NJ, 1995).

${ }^{12}$ G. A. Bird, Molecular Gas Dynamics and the Direct Simulation of Gas Flows (Clarendon, Oxford, 1994).

${ }^{13}$ E. S. Oran, C. K. Oh, and B. Z. Cybyk, Annu. Rev. Fluid Mech. 30, 403 (1998). 